\title{
A GENERAL UNIQUENESS THEOREM
}

ROBERT D. MOYER

1. Introduction. In the past seventy years there has been a large development of various conditions which guarantee the uniqueness of solutions of ordinary differential equations. Many of these conditions are apparently unrelated. In 1949, LaSalle [6] gave two different conditions which covered several different criteria for uniqueness $[2],[3],[4],[5]$. Since then, further uniqueness results appeared which cannot be obtained from LaSalle's conditions [8], [9]. The purpose of this paper is to generalize the various conditions and to unify the methods of proving uniqueness.

The crucial point in the unification is Theorem 2.1. In practice, however, this theorem has little value. Therefore, in Theorem 2.2 we give five different conditions each of which is sufficient for uniqueness. These conditions are generalizations of known conditions (see §3). Finally, on the basis of these generalizations, we shall give a simple uniqueness condition which is compatible with the Carathéodory existence theorem.

2. The basic theorem. In this section we shall be concerned with an ordinary differential equation in a normed linear space $S$. For $y \in S,|y|$ will denote the norm of $y$.

Consider a domain $D$ in $[0,1] \times S$ which projects onto $[0,1]$ and let $f$ be a mapping from $D$ into $S$. We shall be concerned with the problem of finding a differentiable function $y$ such that

$$
\begin{aligned}
y^{\prime}(x) & =f(x, y(x)), \quad x \in[0,1], \\
y(0) & =\alpha,
\end{aligned}
$$

where $\alpha \in S$ and is such that $(0, \alpha) \in D$. Assuming that (2.1) has a solution, we shall seek conditions under which it is the only one.

Now, on the basis of the known uniqueness theorems, it seems reasonable to make the following

Assumption. There is a continuously differentiable function $W(x, r)$, $0<x \leqq 1,0<r<\infty$, such that

(i) $\partial W / \partial x(x, r) \leqq 0, \partial W / \partial r(x, r) \geqq 0,0<x \leqq 1,0<r<\infty$;

(ii) $\partial W / \partial r(x,|y-z|)|f(x, y)-f(x, z)| \leqq-\partial W / \partial x(x,|y-z|), 0<x$ $\leqq 1, y \neq z,(x, y) \in D$, and $(x, z) \in D ;$ and

(iii) $W\left(x, 0^{+}\right)=-\infty, 0<x \leqq 1$.

Received by the editors October 27, 1965. 
By defining $W(x, 0)=-\infty, 0<x \leqq 1$, we may assume that $W$ is a continuous function from $(0,1] \times[0, \infty)$ in to $[-\infty, \infty)$.

REMARK. Although $\partial W / \partial r$ is allowed to be zero for some $r, \partial W / \partial r$ cannot be identically zero in a neighborhood of zero without (iii) being violated. Thus (ii) imposes a condition on the behavior of $|f(x, y)-f(x, z)|$ as $|y-z| \rightarrow 0$.

The remarkable consequence of the above assumption is the following

THEOREM 2.1. Let $y$ and $z$ be two solutions of (2.1). Then $y \equiv z$ if and only if

$$
\operatorname{Liminf}_{x \rightarrow 0^{+}} W(x,|y(x)-z(x)|)=-\infty .
$$

Proof. Let $\phi(x)=|y(x)-z(x)|$ and let

$$
P=\{x \in(0,1): \phi(x)>0\} .
$$

Letting $D^{+}$denote the upper right derivative, we have that

$$
\left|D^{+} \phi(x)\right| \leqq\left|y^{\prime}(x)-z^{\prime}(x)\right|, \quad x \in(0,1) .
$$

From (i) and (ii) it then follows that for $x \in P$,

$$
\begin{aligned}
D^{+} W(x, \phi(x)) & \leqq \frac{\partial W}{\partial x}(x, \phi(x))+\frac{\partial W}{\partial r}(x, \phi(x)) D^{+} \phi(x) \\
& \leqq \frac{\partial W}{\partial x}(x, \phi(x))+\frac{\partial W}{\partial r}(x, \phi(x))\left|y^{\prime}(x)-z^{\prime}(x)\right| \\
& \leqq \frac{\partial W}{\partial x}(x, \phi(x))+\frac{\partial W}{\partial r}(x, \phi(x))|f(x, y(x))-f(x, z(x))| \\
& \leqq 0 .
\end{aligned}
$$

Hence $W(x, \phi(x))$ is nonincreasing on $P$. But $W(x, \phi(x))$ is continuous for $x \in(0,1)$ and $W(x, \phi(x))=-\infty$ when $x \notin P$. Therefore, $W(x, \phi(x))$ is nonincreasing for $x \in(0,1)$. Thus either $P=\varnothing$ or $P=(0, a)$ some $a>0$. Since in the latter case, $W(x, \phi(x))$ would be bounded below near $x=0$, it follows that $\phi \equiv 0$ iff $\operatorname{Lim} \inf _{x \rightarrow 0^{+}} W(x, \phi(x))=-\infty$.

REMARK. In the special case where $W(x, r)=w_{1}(r)-w_{2}(x)$ (which is the case in almost all known uniqueness theorems) the above theorem holds when $w_{1}$ is differentiable on $(0, \infty)$ and $w_{2}$ is absolutely continuous on compact subsets of $(0,1]$. The fact that $W(x, \phi(x))$ is nonincreasing on $P$ in this case has been shown by Heins [1].

Let us now give several sufficient conditions which guarantee uniqueness (or equivalently, (2.2)). 
THEOREM 2.2. Solutions of (2.1) are unique when any one of the following holds:

(a) There is an $r_{0}>0$ such that $W\left(0^{+}, r\right)<\infty, 0<r \leqq r_{0}$, and

$$
\operatorname{Liminf}_{r \rightarrow 0^{+}} W\left(0^{+}, r\right)=-\infty \text {. }
$$

(b) There exists a differentiable function $\omega_{1}(r), 0 \leqq r<\infty$, and an absolutely continuous function $\omega_{2}(x), 0 \leqq x \leqq 1$, such that

$(\alpha) \omega_{1}^{\prime}(r)>0, r \in(0, \infty), \omega_{2}^{\prime}(x) \geqq 0$, a.e. $x \in(0,1)$;

(ß) $\omega_{1}(0)=\omega_{2}(0)=0$;

$(\gamma) \omega_{1}^{\prime}(|y-z|)|f(x, y)-f(x, z)| \leqq \omega_{2}^{\prime}(x)$, a.e. $x \in(0,1), \quad y \neq z$, $(x, y) \in D$, and $(x, z) \in D$;

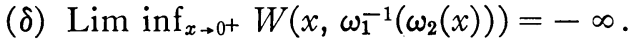

(c) $f$ is continuous on $D$ and $\operatorname{Lim} \inf _{x \rightarrow 0^{+}} W(x, x)=-\infty$.

(d) $f$ is continuous on $D, W(x, x)$ is bounded in a neighborhood of zero, $\partial W / \partial x$ is a nonincreasing function of $r$ and there is an $x_{0}>0$ such that $\operatorname{Lim} \inf _{x \rightarrow 0^{+}}\left[W\left(x_{0}, x h(x)\right)-W\left(x_{0}, x\right)\right]=-\infty$ for every $h$ such that $h(x) \rightarrow 0$ as $x \rightarrow 0^{+}$.

(e) $f$ is continuous and $\operatorname{Lim} \inf _{x \rightarrow 0^{+}} W(x, x h(x))=-\infty$ for every nonnegative function $h$ such that $h(x) \rightarrow 0$ as $x \rightarrow 0^{+}$.

Proof. In what follows, $y$ and $z$ will be two solutions of $(2.1)$ and we shall let $\phi(x)=|y(x)-z(x)|, x \in[0,1]$.

Part (a). By (i), $W$ is nonincreasing in $x$. Thus $W(x, \phi(x)) \leqq$ $W\left(0^{+}, \phi(x)\right), x \in(0,1]$. But $\phi(x) \rightarrow 0$ as $x \rightarrow 0^{+}$. Therefore

$$
\operatorname{Liminf}_{x \rightarrow 0^{+}} W(x, \phi(x)) \leqq-\infty .
$$

Part (b). From the results of Heins [1], it follows that $\omega_{1}(\phi(x))$ $-\omega_{2}(x)$ is a nonincreasing function of $x \in(0,1)$. Since $\phi(0)=0$, we have by $(\beta)$ that $\omega_{1}(\phi(0))-\omega_{2}(0)=0$ and thus $\omega_{1}(\phi(x)) \leqq \omega_{2}(x)$. But, $\omega_{1}$ is increasing and thus $\omega_{1}^{-1}$ exists. Hence, we have that

$$
\phi(x) \leqq \omega_{1}^{-1}\left(\omega_{2}(x)\right), \quad x \in[0,1] .
$$

Since $W(x, r)$ is a nondecreasing function of $r, W(x, \phi(x))$ $\leqq W\left(x, \omega_{1}^{-1}\left(\omega_{2}(x)\right)\right), x \in(0,1]$, and from $(\delta)$ it follows that

$$
\operatorname{Liminf}_{x \rightarrow 0^{+}} W(x, \phi(x)) \leqq-\infty .
$$

Part (c). When $f$ is continuous, we have that $y$ and $z$ are continuously differentiable and that

Thus

$$
\left|y^{\prime}(0)-z^{\prime}(0)\right|=|f(0, \alpha)-f(0, \alpha)|=0 .
$$




$$
\operatorname{Liminf}_{x \rightarrow 0^{+}} \frac{\phi(x)}{x}=0 .
$$

But then $\phi(x) \leqq x$ for all $x$ sufficiently small and we have that

$$
\operatorname{Liminf}_{x \rightarrow 0^{+}} W(x, \phi(x)) \leqq \operatorname{Liminf}_{x \rightarrow 0^{+}} W(x, x)=-\infty .
$$

Part (d). Again we have $\phi(x) / x \rightarrow 0$ as $x \rightarrow 0^{+}$. Also, for $t$ sufficiently small we have that $\phi(t) \leqq t$ and thus $\partial W / \partial x(x, \phi(t)) \geqq \partial W / \partial x(x, t)$. Integrating this inequality from $t$ to $x_{0}$ (where $t \leqq x_{0}$ ) we have

$$
W\left(x_{0}, \phi(t)\right)-W(t, \phi(t)) \geqq W\left(x_{0}, t\right)-W(t, t)
$$

or

$$
\begin{aligned}
W(t, \phi(t)) & \leqq W\left(x_{0}, \phi(t)\right)-W\left(x_{0}, t\right)+W(t, t) \\
& \leqq W\left(x_{0}, t \frac{\phi(t)}{t}\right)-W\left(x_{0}, t\right)+W(t, t) .
\end{aligned}
$$

But $W(t, t)$ is bounded and $h(t)=\phi(t) / t \rightarrow 0$ as $t \rightarrow 0^{+}$. Therefore

$$
\operatorname{Liminf}_{t \rightarrow 0^{+}} W(t, \phi(t)) \leqq-\infty .
$$

\begin{tabular}{|c|c|c|c|}
\hline$W(x, r)$ & Assumptions & & Originator \\
\hline 1. $\log r-K x$ & & a & Lipschitz \\
\hline 2. $w_{1}(r)-K x$ & $w_{1}\left(0^{+}\right)=-\infty$ & a & Osgood [2] \\
\hline 3. $w_{1}(r)-w_{2}(x)$ & $w_{1}\left(0^{+}\right)=-\infty,\left|w_{2}\left(0^{+}\right)\right|<\infty$ & $\mathrm{a}$ & $\begin{array}{l}\text { Montel [3] } \\
\text { Heins [1] }\end{array}$ \\
\hline 4. $\log r-\beta \log x$ & $0<\beta<1$ & c & Rosenblatt [4] \\
\hline 5. $\log r-\log x$ & & $\mathrm{e}$ & Nagumo [5] \\
\hline 6. $w_{1}(r)-w_{2}(x)$ & $w_{1}(x)-w_{2}(x) \rightarrow-\infty$ as $x \rightarrow 0^{+}$ & $\mathrm{c}$ & LaSalle [6] \\
\hline 7. $w_{1}(r)-w_{2}(x)$ & $\begin{array}{l}w_{1}(x)-w_{2}(x) \text { bounded and } w_{1}(x h(x)) \\
-w_{2}(x) \rightarrow-\infty \text { for those } h \geqq 0 \text { such } \\
\text { that } h(x) \rightarrow 0 \text { as } x \rightarrow 0^{+}\end{array}$ & $\mathrm{d}$ & LaSalle [6] \\
\hline 8. $\log r-K \log x$ & $\omega_{1}=r^{1-\alpha}, \omega_{2}=p x ; 0<1-\alpha<1 / k$ & $\mathrm{~b}$ & $\begin{array}{l}\text { KrasnoselskiY } \\
\text { and KreIn [7] }\end{array}$ \\
\hline 9. $w_{1}(r / x)-w_{2}(x)$ & $w_{1}\left(0^{+}\right)=-\infty,\left|w_{2}\left(0^{+}\right)\right|<\infty$ & e & Walter [8] \\
\hline
\end{tabular}

Part (e). Again $\phi(x) / x \rightarrow 0$ as $x \rightarrow 0^{+}$. Thus

$$
\operatorname{Liminf}_{x \rightarrow 0^{+}} W(x, \phi(x))=\operatorname{Liminf}_{x \rightarrow 0^{+}} W\left(x, x \frac{\phi(x)}{x}\right)=-\infty .
$$

3. Examples. In the table below we list the known conditions which guarantee uniqueness. The letter in column three indicates which part of Theorem 2.2 is applicable. 
4. Carathéodory problem. Consider the special case where $S=R^{n}$. In this case let us replace $(2.1)$ by the problem of finding an absolutely continuous function $y$ on $[0,1]$ such that

$$
\begin{aligned}
y^{\prime}(x) & =f(x, y(x)), \text { a.e. } x \in(0,1), \\
y(0) & =\alpha .
\end{aligned}
$$

As before, we shall assume that (4.1) has a solution and ask if there is only one.

By again assuming that there is a continuous function $W(x, r)$, such that for each $r \in(0, \infty) W$ is an absolutely continuous function of $x$ on compact subsets of $(0,1]$, such that $(\partial W / \partial r)(x, r)$ exists and is continuous on $(0,1] \times(0, \infty)$, and such that $W$ satisfies (i)-(iii), we have

THEOREM 4.1. If $y$ and $z$ are two absolutely continuous solutions of (4.1), then $y \equiv z$ iff

$$
\operatorname{Liminf}_{x \rightarrow 0^{+}} W(x,|y(x)-z(x)|)=-\infty .
$$

Proof. Let $\phi=|y-z|$ and $P=\{x \in(0,1): \phi(x)>0\}$. Since $S=R^{n}$, we have that $\phi^{\prime}(x)$ exists and $\left|\phi^{\prime}(x)\right| \leqq\left|y^{\prime}(x)-z^{\prime}(x)\right|$ for a.e. $x \in P$. Thus

$$
\begin{aligned}
\frac{d}{d x} W(x, \phi(x)) & =\frac{\partial W}{\partial x}(x, \phi(x))+\frac{\partial W}{\partial r}(x, \phi(x)) \phi^{\prime}(x) \\
& \leqq 0
\end{aligned}
$$

for a.e. $x \in P$. Also, $W(x, \phi(x))$ is an absolutely continuous function on compact subsets of $(0,1]$. Thus $W(x, \phi(x))$ is a nonincreasing function of $x$ and we have the desired result as in the proof of Theorem 2.1 .

Clearly we have

THEOREM 4.2. If $W$ satisfies any of the conditions of Theorem 2.2, then solutions of (4.1) are unique.

Our main interest here is to state a fairly simple condition which gives the uniqueness of solutions of (4.1) whose existence are guaranteed by the existence theorem of Carathéodory. We have

THEOREM 4.3. Let $f$ be measurable in $x$ for each $y$ and continuous in $y$ for each $x$. Assume there is an integrable function $m(x), x \in(0,1)$ such that

$$
|f(x, y)| \leqq m(x) \text {, a.e. } x \in(0,1) \text {. }
$$


Finally, letting $M(x)=\int_{0}^{x} m(\xi) d \xi$ and assuming that

$$
\operatorname{Liminf}_{x \rightarrow 0^{+}} W(x, 2 M(x))=-\infty,
$$

we have that there exists one and only one solution of (4.1).

Proof. Existence is guaranteed by the Carathéodory theorem (see [9]). Now, let $\omega_{1}(r)=r$ and $\omega_{2}(x)=2 M(x)$. Then

$$
\begin{aligned}
\omega_{1}^{\prime}(|y-z|)|f(x, y)-f(x, z)| & =|f(x, y)-f(x, z)| \\
& \leqq 2 m(x) \\
& \leqq \omega_{2}^{\prime}(x) .
\end{aligned}
$$

Thus by Theorems $2.2 \mathrm{~b}$ and 4.2 , we have that solutions of 4.1 are unique.

\section{BIBLIOGRAPHY}

1. Maurice Heins, Some remarks on unicity and continuity theorems for ordinary differential equations, Michigan Math. J. 10 (1963), 85-89.

2. W. Osgood, Beweise der Existenz einer Lösung der Differentialgleichungen $d y / d x=f(x, y)$ ohne Hinzunahme der Cauchy-Lipschitz Bedingung, Monatsh. Math. Physik 9 (1898), 331-345.

3. P. Montel, Sur l'intégrale supérieure et l'intégrale inférieure d'une équation différentielle, Bull. Sci. Math. (2) 50 (1926), 205-217.

4. A. Rosenblatt, Über die Existenz von Integralen gewöhnlicher Differentialgleichungen, Arch. Math. Astronom. Fys. 5 (1909), no. 2.

5. M. Nagumo, Eine hinreichende Bedingung für die Unität der Lösung von Differentialgleichungen erster Ordnung, Japan J. Math. 3 (1926), 107-112.

6. J. LaSalle, Uniqueness theorems and successive approximations, Ann. Math. 50 (1949), 722-730.

7. M. A. Krasnoselskir and S. G. KreIn, On a class of uniqueness theorems for the equation $y^{\prime}=f(x, y)$, Uspehi Mat. Nauk (67) 11 (1956), 209-213. (Russian)

8. W. Walter, Eindeutigkeitssätze für gewöhnliche, parabolische und hyperbolische Differentialgleichungen, Math. Z. 74 (1960), 191-209.

9. E. A. Coddington, and N. Levinson, Theory of ordinary differential equations, McGraw-Hill, New York, 1955.

Pennsylvania State University 